\title{
Right-sided Bochdalek hernia in an adult with hepatic malformation and intestinal malrotation
}

\author{
Naoki Enomoto, Kazuhiko Yamada* (1), Daiki Kato, Shusuke Yagi, Hitomi Wake, Kyoko Nohara, \\ Nobuyuki Takemura, Tomomichi Kiyomatsu and Norihiro Kokudo
}

\begin{abstract}
Background: Bochdalek hernia is a common congenital diaphragmatic defect that usually manifests with cardiopulmonary insufficiency in neonates. It is very rare in adults, and symptomatic cases are mostly left-sided. Diaphragmatic defects generally warrant immediate surgical intervention to reduce the risk of incarceration or strangulation of the displaced viscera.

Case presentation: A 47-year-old woman presented with dyspnea on exertion. Computed tomography revealed that a large part of the intestinal loop with superior mesenteric vessels and the right kidney were displaced into the right thoracic cavity. Preoperative three-dimensional (3D) simulation software visualized detailed anatomy of displaced viscera and the precise location and size of the diaphragmatic defect. She underwent elective surgery after concomitant pulmonary hypertension was stabilized preoperatively. The laparotomic approach was adopted. Malformation of the liver and the presence of intestinal malrotation were confirmed during the operation. The distal part of the duodenum, jejunum, ileum, colon, and right kidney were reduced into the abdominal cavity consecutively. A large-sized oval defect was closed with monofilament polypropylene mesh. No complications occurred postoperatively.
\end{abstract}

Conclusion: Symptomatic right-sided Bochdalek hernia in adults is exceedingly rare and is frequently accompanied by various visceral anomalies. Accurate diagnosis and appropriate surgical repair are crucial to prevent possible incarceration or strangulation. The preoperative 3D simulation provided comprehensive information on anatomy and concomitant anomalies and helped surgeons plan the operation meticulously and perform procedures safely.

Keywords: Bochdalek hernia, Adult, Right-sided, Hepatic malformation, Intestinal malrotation, Three-dimensional simulation

\section{Introduction}

Bochdalek hernia is a diaphragmatic hernia located on the posterolateral portion of the diaphragm [1]. Most cases are left-sided and are commonly diagnosed during the neonatal period. Neonatal Bochdalek hernia frequently compromises the cardiopulmonary function and carries a high rate of mortality.

Right-sided Bochdalek hernia is extremely rare in adults. Early closure of the right pleuroperitoneal canal and the buttressing effect of the liver may explain the reason [2]. We present an adult case of right-sided Bochdalek hernia accompanied by malformation of the liver and intestinal malrotation.

*Correspondence: kayamada@hosp.ncgm.go.jp

Department of Surgery, Center Hospital of the National Center for Global

Health and Medicine, 1-21-1 Toyama, Shinjuku-ku, Tokyo 162-8655, Japan 


\section{Case presentation}

The patient was a 47-year-old woman who presented with dyspnea on exertion. Her medical history was remarkable for asthma and ovarian cyst. At regular health check-ups during her elementary school years, chest radiographs appeared abnormal; however, she never had any opportunities for further examinations until this presentation.

Chest radiographs showed a massive area of opacity mixed with intestinal gas in the right lung field (Fig. 1). Computed tomography (CT) scans revealed that both the right kidney and the portion of the alimentary tract from the distal part of the duodenum to the transverse colon had herniated into the right thoracic cavity (Fig. 2). A cardiac catheterization study demonstrated elevated pulmonary capillary wedge pressure $(31 \mathrm{~mm} \mathrm{Hg})$ and elevated pulmonary arterial pressure $(53 \mathrm{~mm} \mathrm{Hg})$. Rightsided Bochdalek hernia and pulmonary hypertension were diagnosed. After her cardiopulmonary function was stabilized with medical therapy, she was referred to the surgical department for operative repair of the diaphragmatic hernia.

To visualize the anatomy and vasculature preoperatively, we used 3D analysis software (Synapse Vincent; Fujifilm, Tokyo, Japan) to generate three-dimensional (3D) images from thin-section reconstructed CT data (Fig. 3). These images showed that the superior mesenteric artery (SMA) and vein (SMV) had herniated through the diaphragmatic defect. The eleventh and twelfth ribs with intercostal muscle constituted the posterolateral part of the defect. The size of the elliptical defect was estimated to be $11 \times 5 \mathrm{~cm}$. The liver was flattened in shape, which left the large diaphragmatic defect uncovered. The right kidney was detached from the retroperitoneum and had also herniated through the defect.

A transabdominal approach was selected given the difficulty in reducing the huge volume of herniated contents through the transthoracic approach. We adopted a laparotomic approach, because reducing the main trunks of SMA and SMV laparoscopically might have been unsafe, and any resulting pneumoperitoneum might have worsened the preexisting cardiopulmonary burden.

The patient was placed supine while under general anesthesia, and a J-shaped incision was made in the abdomen. The flattened appearance of the liver was confirmed. We fully mobilized the right lobe of the liver to expose the diaphragm. The small intestine and ascending colon were attached to the common mesentery without any retroperitoneal fixation. We confirmed intestinal malrotation (IM) and the absence of the ligament of Treitz (Fig. 4). The IM was classified as a nonrotation pattern.

We gently dissected the adhesion between the intestines and the rim of the defect. First, the colon was pulled back to the abdominal cavity, and then the distal part of the duodenum, ileum, and jejunum were sequentially reduced. There was neither adhesion nor hernia sac in the thoracic cavity. We meticulously manipulated the main trunks of SMA and SMV during their reduction. The defect was completely exposed following the reduction of
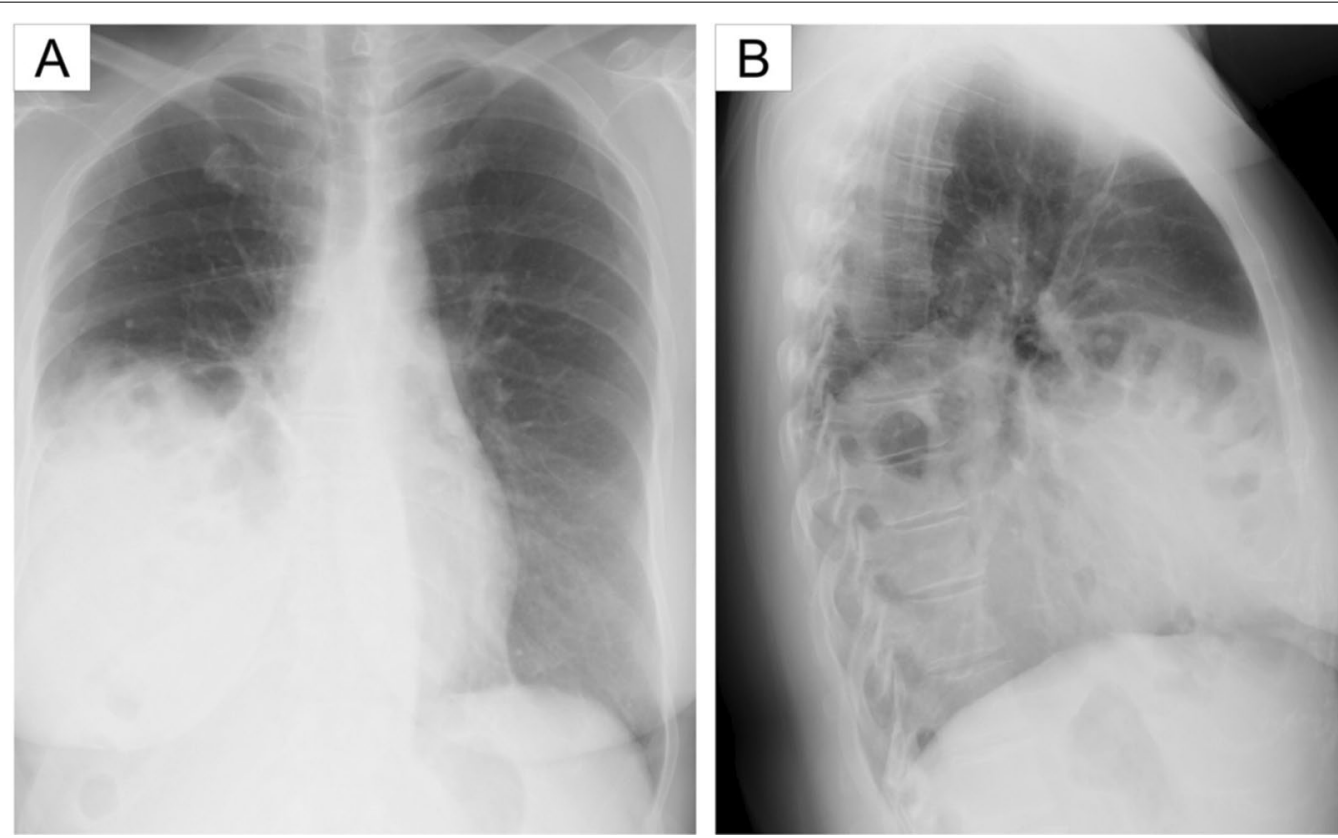

Fig. 1 Preoperative chest radiography. a Frontal chest radiography showed a massive area of opacity in the right lung field. $\mathbf{b}$ Lateral chest radiography showed intestinal gas in opacity in the lung fields 


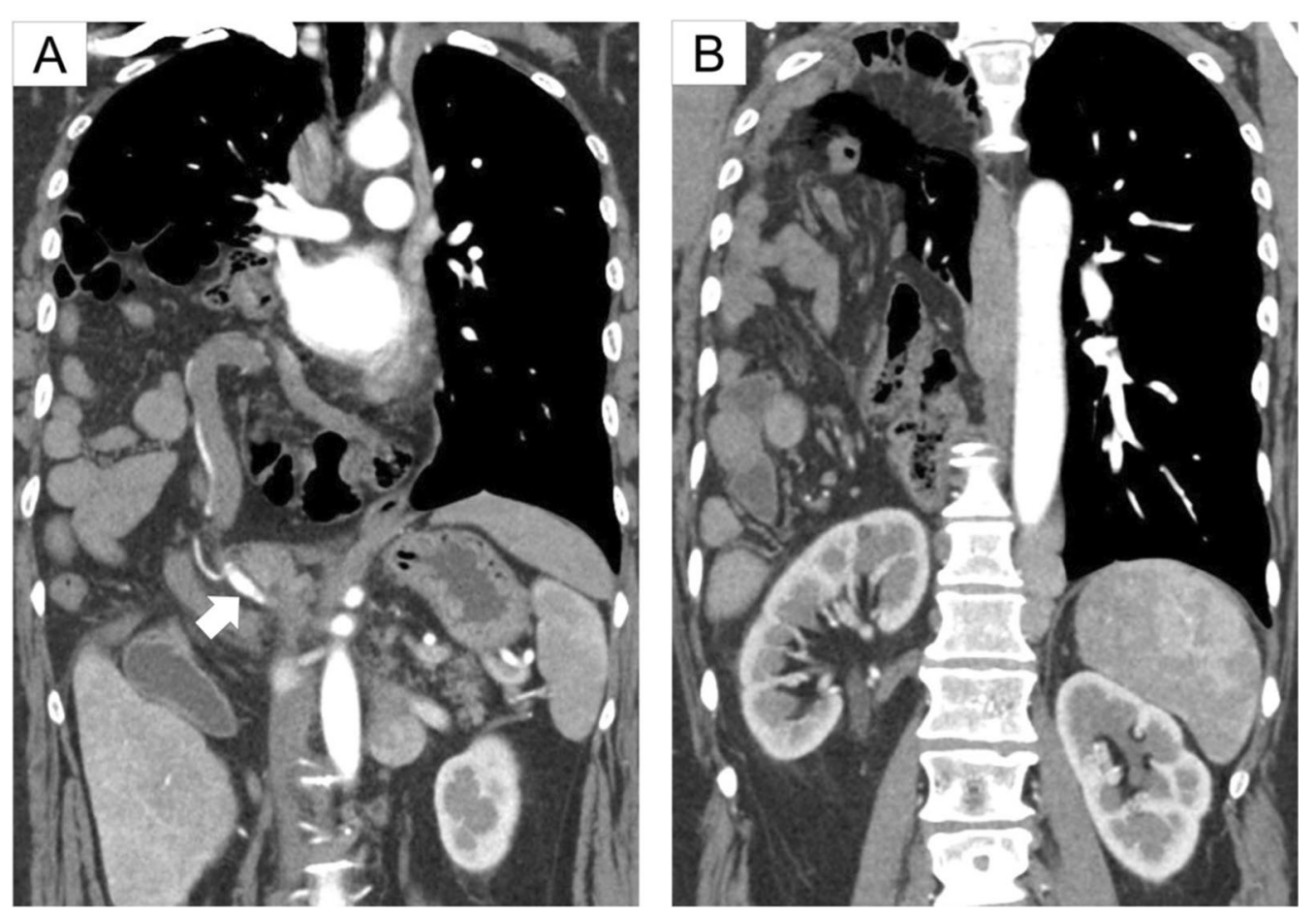

Fig. 2 Coronal views of preoperative contrast medium-enhanced thoracoabdominal computed tomographic scans. a Small intestine, the colon, and the main trunk of the superior mesenteric artery (white arrow) had herniated through the diaphragmatic defect into the right thoracic cavity. $\mathbf{b}$ Right kidney was detached from the retroperitoneum and had also herniated into the right thoracic cavity

the right kidney. The diameter of the defect was indeed $11 \times 5 \mathrm{~cm}$, as estimated. Monofilament polypropylene mesh (Ventralight ${ }^{\mathrm{TM}}$ ST mesh; Becton, Dickinson and Company, Franklin Lakes, NJ, USA) was cut in an oval with sufficient margins and fixed to the diaphragm circumferentially (Fig. 5). Intestinal repositioning or fixation was not performed, because nonrotation pattern IM has a low risk of intestinal volvulus.

The patient was discharged without any complications on postoperative day 10. Dyspnea on exertion was relieved, and the severity of pulmonary hypertension gradually lessened over time. The pulmonary capillary wedge pressure and mean pulmonary arterial pressure decreased to 10 and $20 \mathrm{~mm} \mathrm{Hg}$, respectively. She exhibited no recurrence of the hernia at the 20-month followup visit (Fig. 6).

\section{Discussion}

By the eighth week of embryonic development, the pleuroperitoneal membranes have fused with the septum transversum centrally, the mesoesophagus mediodorsally, and the musculature of the lateral body wall $[1,3]$. Failure to fuse leads to several types of diaphragmatic hernia. Failure of the septum transversum to fuse anteriorly leads to Morgagni hernias. Posterolateral defects
(Bochdalek hernias) were first described by Victor Alexander Bochdalek in 1848. Bochdalek hernia occurs as the consequence of the incomplete fusion of the pleuroperitoneal membrane, the dorsal esophageal mesentery, and the body wall [4].

Most cases of Bochdalek hernia with large defects are diagnosed during the neonatal period due to the accompanying severe respiratory failure. Congenital Bochdalek hernia is the most common congenital diaphragmatic hernia $(\mathrm{CDH})$, accounting for $90 \%$ to $95 \%$ of such hernias $[5,6]$. The incidence of congenital Bochdalek hernia has been estimated to be 1 per 2000 to 12,500 live births, and $85 \%$ of congenital Bochdalek hernias are left-sided [2].

Bochdalek hernia is rarely found in adults. It could occur after trauma, pregnancy, abdominal surgery, and other events that increase intra-abdominal pressure and exacerbate subtle diaphragmatic defects that are too small to cause symptoms early in life. Asymptomatic adult Bochdalek hernia is usually found incidentally with imaging modalities, such as chest X-ray or multidetector-row CT. In a review of 13,138 abdominal CT scans performed to rule out metastatic disease in patients with known malignancy, the incidence of asymptomatic adult Bochdalek hernia was $0.17 \%$; the defect was right-sided in $68 \%$ of the affected patients, 

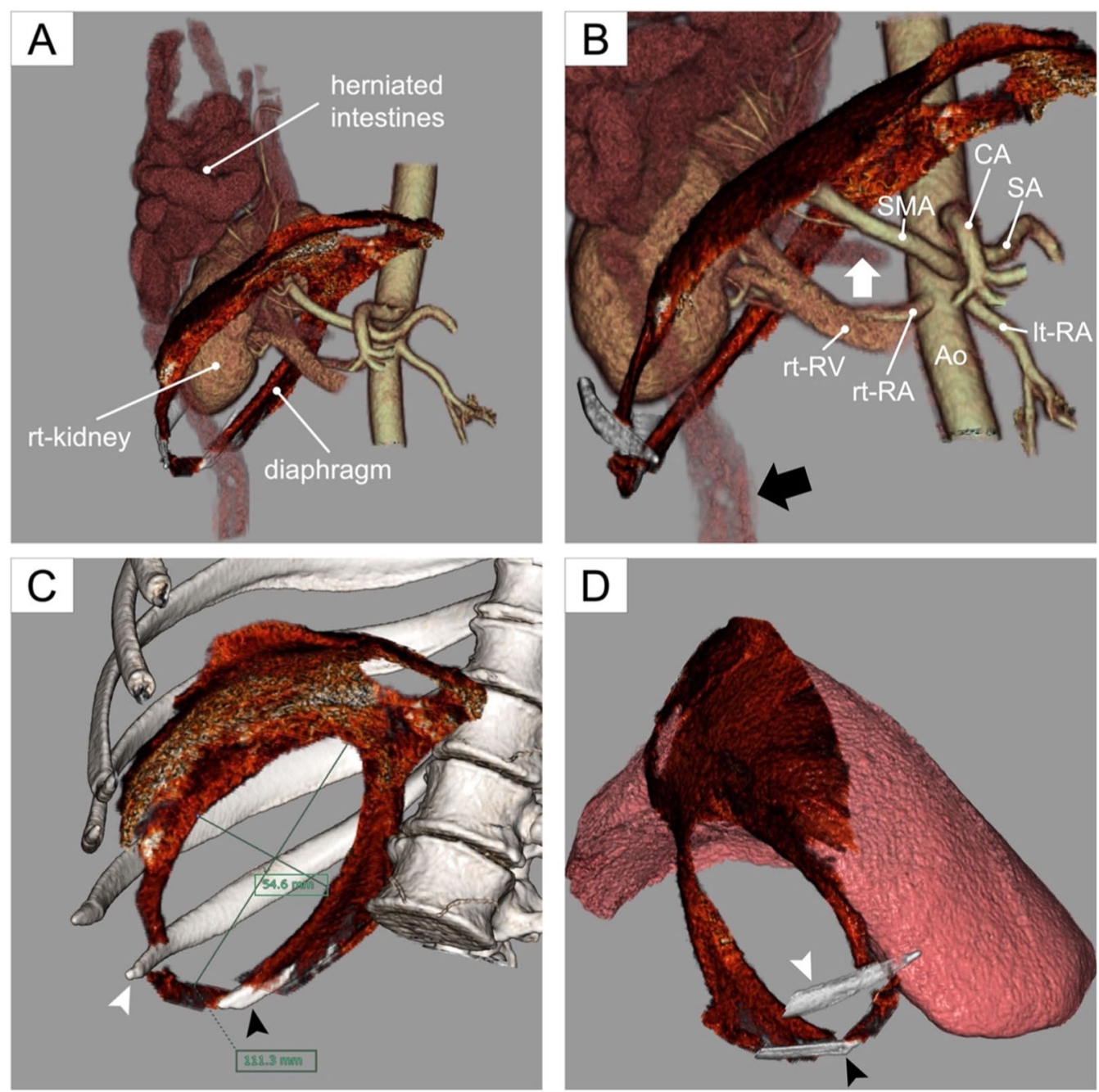

Fig. 3 Preoperative three-dimensional (3D) simulation. a Herniated intestines and the right kidney occupied the right thoracic cavity. b Portion of the alimentary tract [from the distal part of duodenum (white arrow) to the proximal part of transverse colon (black arrow)] and the main trunk of superior mesenteric artery had herniated through the diaphragmatic defect. $\mathbf{c}$ Oval diaphragmatic defect was present on the posterolateral portion of the diaphragm. 3D simulation precisely visualized the location and size of the defect. The eleventh rib (white arrowhead) and the twelfth rib (black arrowhead) with intercostal muscles constituted the posterolateral part of the defect. $\mathbf{d}$ Right lobe of the liver was flattened, thus leaving the large diaphragmatic defect uncovered. Ao aorta, CA celiac artery, It-RA left renal artery, rt-kidney right kidney, rt-RA right renal artery, rt-RV right renal vein, SA splenic artery, SMA superior mesenteric artery

and $77 \%$ of the affected patients were female [7]. Of note, asymptomatic cases of adult Bochdalek hernia were found more on the right side than on the left side, whereas congenital Bochdalek hernia is generally found on the left side. However, of 109 adult patients with symptomatic Bochdalek hernia who underwent surgical repair, $82 \%$ had left-sided defects [8]. A buttressing effect of the liver that prevents the displacement of abdominal viscera into the thoracic cavity may explain the difference in laterality between asymptomatic and symptomatic Bochdalek hernia in adults. Right-sided Bochdalek hernia in adults is extremely rare. Through an extensive search with the Japan Medical Abstracts Society and PubMed, we identified and analyzed 43 case reports concerning right-sided Bochdalek hernia in adults. Cases without surgical repair and operative details and the case reports without Japanese or English translations were excluded. These are summarized in Table 1 . The median age was 64 years, and 22 patients (65\%) were female. The median size of the diaphragmatic defect was $7 \mathrm{~cm}$ in diameter. Twenty patients (57\%) presented with abdominal or gastrointestinal symptoms, while 17 cases (49\%) had chest or respiratory symptoms. As to surgical routes, 23 cases $(68 \%), 3$ 


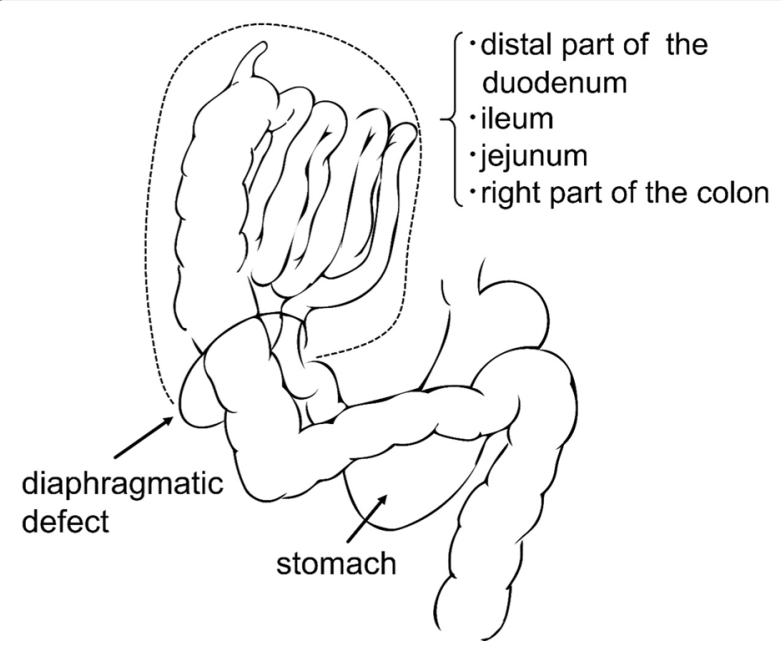

Fig. 4 Schematic illustration of the herniated digestive tract and intestinal malrotation. The distal part of the duodenum, ileum, jejunum, and the right part of the colon were herniated into the right thoracic cavity. The duodenum, cecum, and the ascending colon had lost the fixation to the retroperitoneum

cases (9\%), and 8 cases (23\%) had abdominal, thoracic, and combined thoracoabdominal approaches, respectively. Seven patients underwent minimally invasive surgery. Mesh repair, suture repair, and the combined method were selected in 10 cases (29\%), 22 cases $(65 \%)$, and 2 cases $(6 \%)$, respectively.

Bochdalek hernia is known to accompany several types of congenital anomalies, such as mesenterium commune, situs inversus, intestinal malrotation (IM), gastric volvulus, and malformation of the liver [9]. IM is a congenital anomaly that results from an abnormal rotation of the midgut. It occurs as the midgut returns to the abdominal cavity during the ninth to tenth week of gestation. The incidence of symptomatic IM is estimated to be 1 in 500 live births [10]. IM is classified into four patterns; normal, incomplete, reverse, and nonrotation based on the presumed timing of the developmental failure [11]. The patient had a nonrotation pattern of IM in this presentation. The nonrotation pattern is the most frequent in adults and is caused by the failure to complete the final rotation of $180^{\circ}$ following the normal first $90^{\circ}$ rotation [12]. Nonrotation pattern IM has a low risk of volvulus; thus intestinal repositioning is not necessary in cases without intestinal obstruction [13].

Hypoplasia or atrophy of the right lobe of the liver can facilitate herniation of abdominal organs through the uncovered defect and is frequently observed in right-sided Bochdalek hernia. Malformation of the right lobe of the liver may be the consequence of compromised portal perfusion owing to the long-standing compression effect of displaced organs.
Common symptoms of Bochdalek hernia in adults include dyspnea, cough, chest pain, abdominal pain, and vomiting [2]. Bochdalek hernia in adults is often difficult to diagnose, because the symptoms are often mild and nonspecific $[3,13]$. The use of appropriate imaging modalities, such as magnetic resonance imaging or $\mathrm{CT}$, is crucial for accurate diagnosis. If diagnosis and treatment are delayed, incarceration or strangulation of herniated organs can occur $[14,15]$. Surgical treatment is recommended regardless of symptoms or laterality, in view of the potential risk of visceral complications $[16,17]$.

Bochdalek hernia is surgically repaired with a thoracic, abdominal, or combined thoracoabdominal approach [8]. The advantages of the thoracic approach over the abdominal approach are direct visualization of herniated contents and nonnegative intrapleural pressure, which facilitates the reduction [18]. Through the thoracic approach, adhesions between the displaced viscera and lungs or pleurae can be safely dissected, although few intrapleural adhesions were found in previous reports. In contrast, the advantage of the abdominal approach is easier recognition and management of possible intestinal strangulation and concomitant anomalies of abdominal organs [19]. The abdominal approach is more popular than the thoracic approach and might be suitable in cases complicated by potential strangulation, ischemia, or visceral anomalies. Minimally invasive surgeries with thoracoscopic, laparoscopic, and robotic procedures are increasingly used for Bochdalek hernia on both sides recently [19-23].

The diaphragmatic defect is usually closed with sutures for minor defects. The use of mesh might be recommended for cases with large defects or fragile diaphragm texture, or patients who are extremely obese. We selected mesh repair, considering the large size of the defect and the obese body shape in the present case.

Giant abdominal hernia is associated with postoperative abdominal compartment syndrome (ACS) following the reduction of herniated contents. The use of appropriate mesh, resection of intestines to lessen the volume of the abdominal organs, and delayed abdominal closure are measures to decrease the risk of ACS. ACS after repair of $\mathrm{CDH}$ in neonates is frequently reported [24]. Delayed abdominal closure with the use of silicone silo bags or vacuum-assisted closure devices is performed to prevent ACS in neonates with a large volume of herniated content and limited abdominal capacity. In contrast, ACS after repair of diaphragmatic hernia in adults is extremely rare though few cases were reported. The use of sufficiently large-sized mesh, the adequate volume of the abdominal cavity, and the absence of excessive wound tension might contribute to the uneventful course postoperatively in this case. 

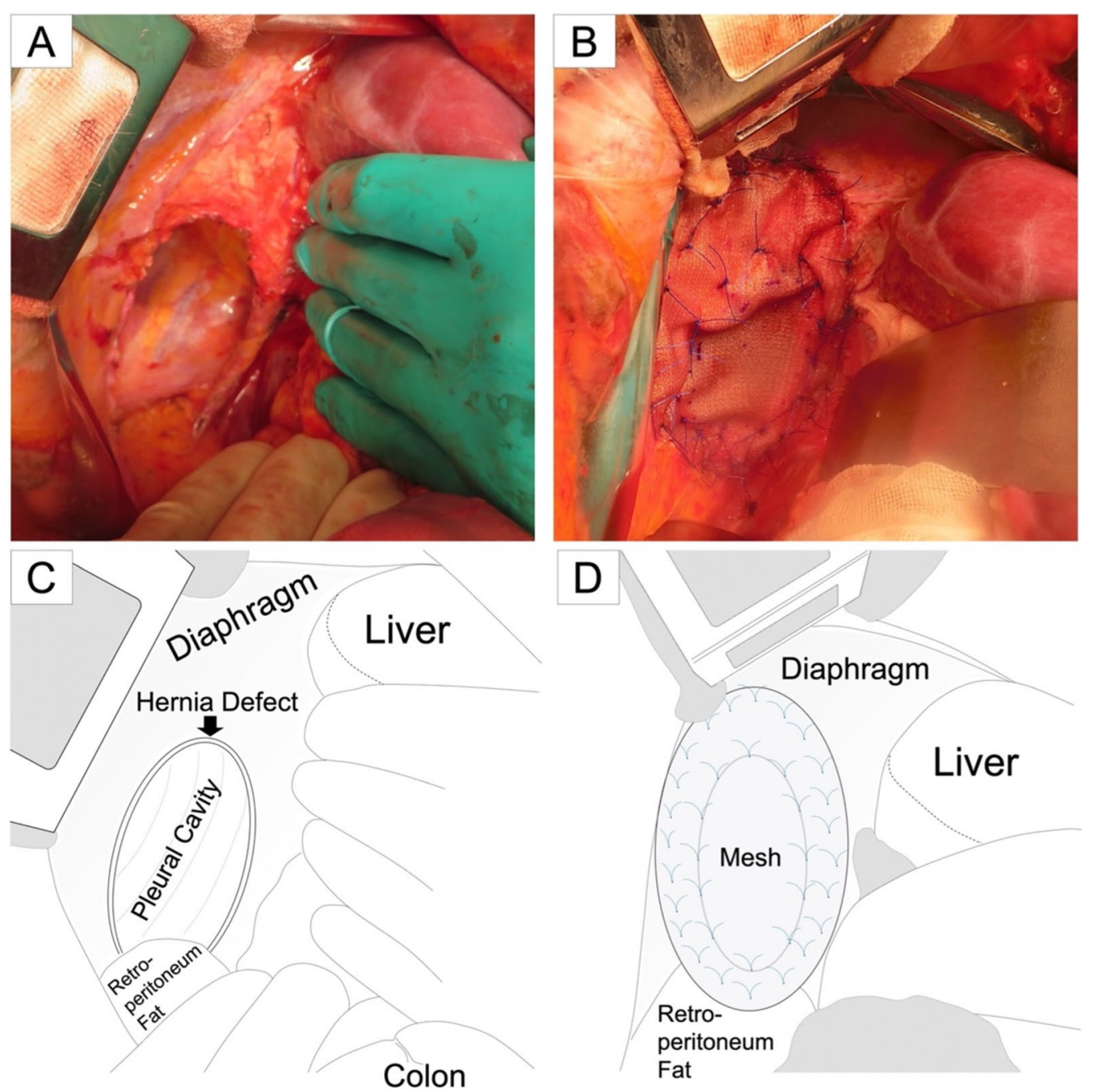

Fig. 5 Intraoperative photographs. a Distal part of the duodenum, ileum, jejunum, the right part of the colon, and the right kidney were reduced into the abdominal cavity consecutively. The defect was exposed on the posterolateral portion of the diaphragm, as predicted. $\mathbf{b}$ Large oval defect was closed with monofilament polypropylene mesh. c Schematic illustration of the photograph A. d Schematic illustration of the photograph B
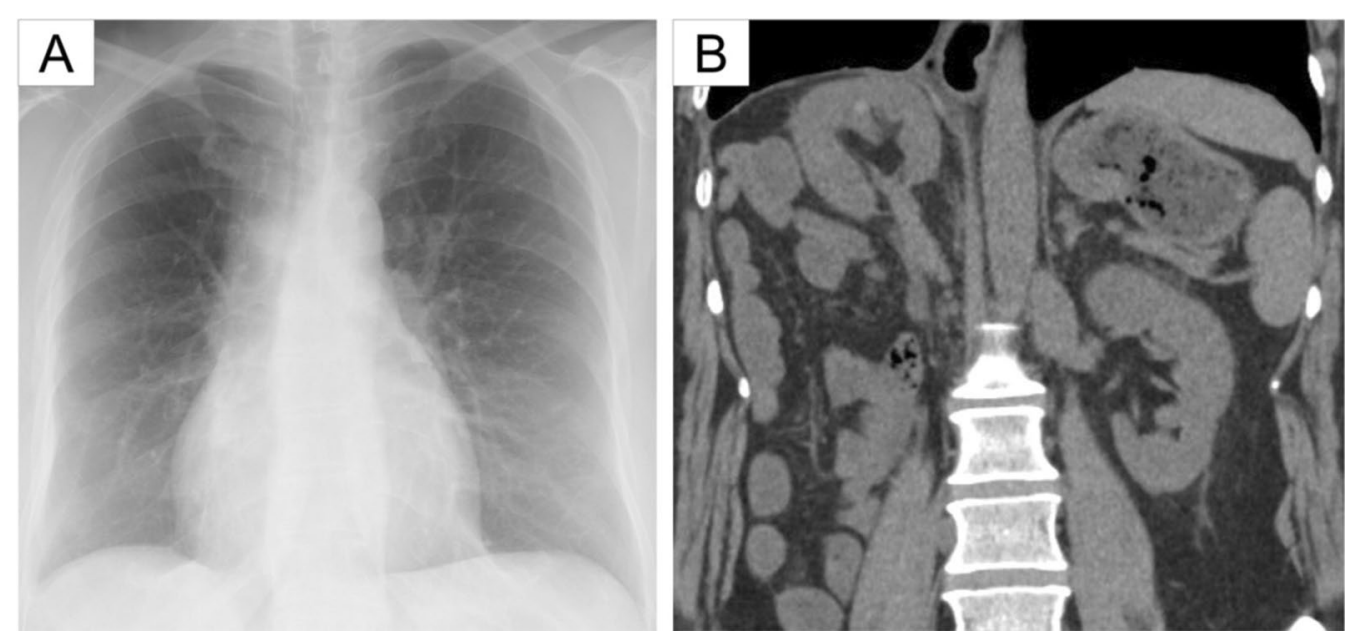

Fig. 6 Postoperative chest radiograph (a) and coronal abdominal computed tomographic (CT) scan (b). a Massive area of opacity had completely disappeared. $\mathbf{b}$ No recurrence of the diaphragmatic hernia was confirmed 20 months after the operation 


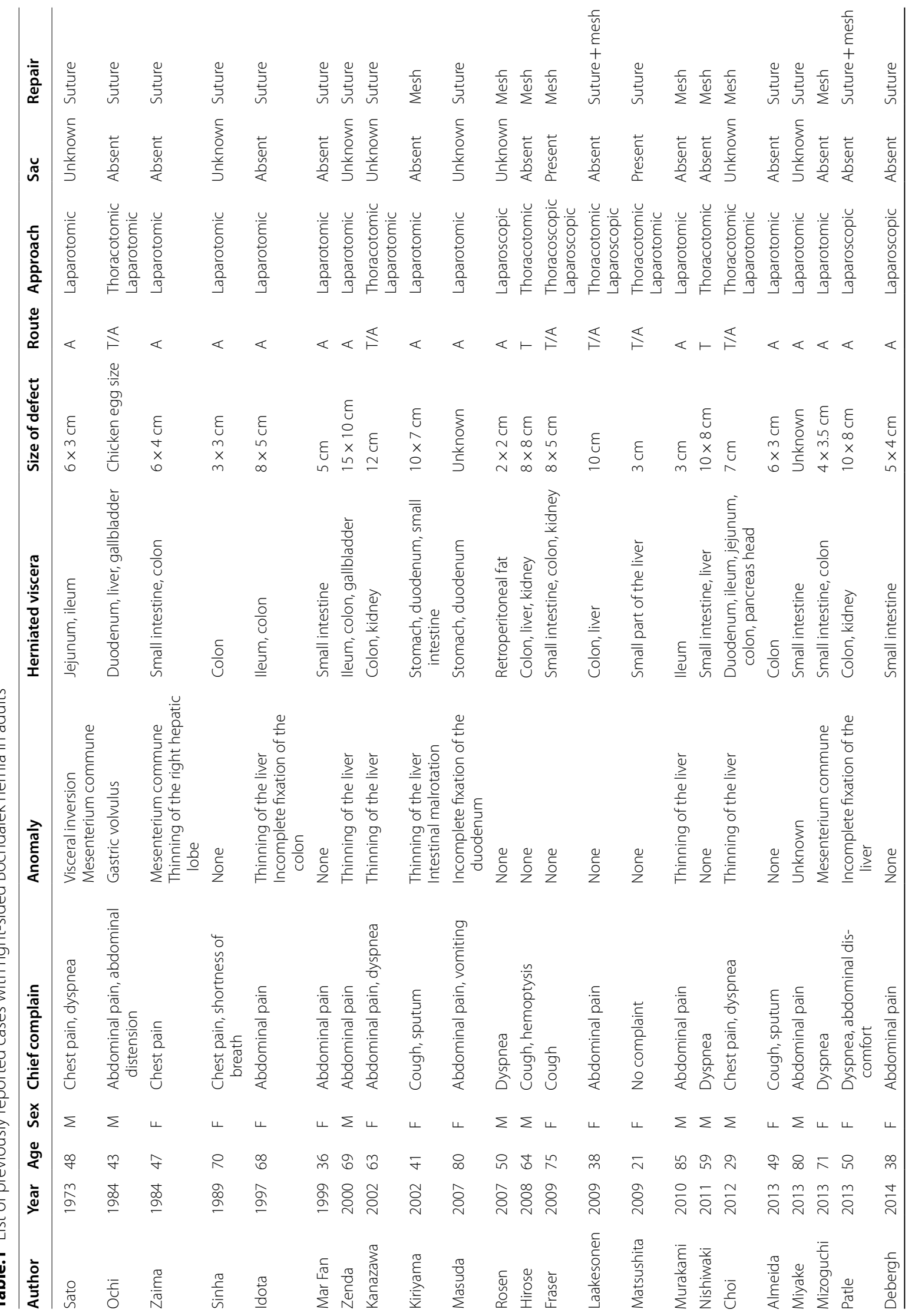




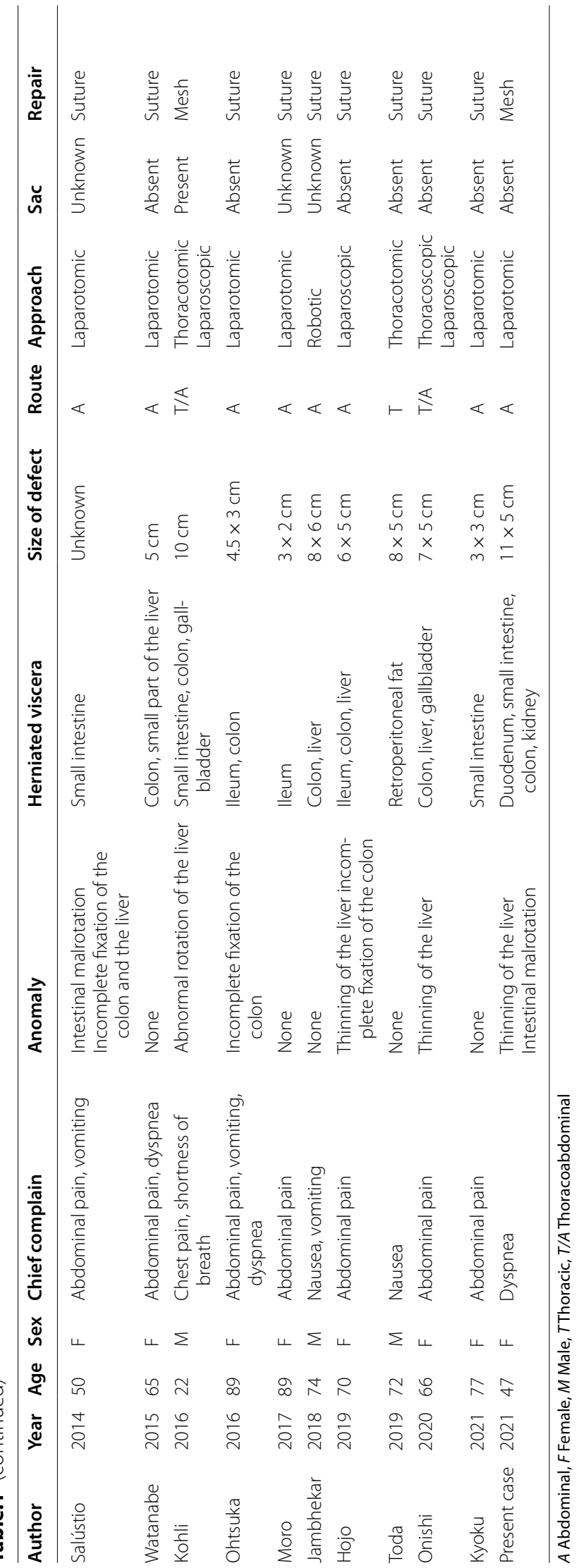


$\mathrm{CDH}$ is associated with respiratory distress and pulmonary hypertension because of pulmonary hypoplasia in neonates [25]. Persistent pulmonary hypertension (PPH) after successful repair of $\mathrm{CDH}$ is one of the significant risk factors for morbidity and mortality in infants [26]. The patient had the potential to develop PPH following the surgical repair of Bochdalek hernia, because she had preoperative pulmonary hypertension in the present case. Fortunately, pulmonary capillary wedge pressure and pulmonary arterial pressure gradually decreased within acceptable range after surgery over time.

3D simulation software has been developed to depict anatomical structures and has assisted surgeons in performing precise and safe procedures since the late 1990s [27]. Virtual reality or augmented reality modalities based on 3D data navigate a broad variety of procedures [28-32]. Few prior studies have demonstrated the usefulness of 3D simulation for diaphragmatic hernia surgery. In this case, the visualization of the displaced viscera and vessels, the deformed liver, and the diaphragmatic defect allowed us to prepare the appropriate mesh and perform safe procedures without any postoperative complications.

\section{Conclusions}

Bochdalek hernia is the most common type of $\mathrm{CDH}$ and generally manifests during the neonatal period. Right-sided Bochdalek hernia in adults is extremely rare, and various congenital visceral anomalies accompany most cases. Accurate diagnosis and appropriate surgical repair are crucial for preventing incarceration or strangulation of abdominal organs. Preoperative 3D simulation offers detailed information about the herniated viscera and vessels, the diaphragmatic defect, and concomitant anomalies. We had developed a meticulous operative plan based on the simulation and successfully performed surgical repair safely.

\section{Abbreviations \\ ACS: Abdominal compartment syndrome; $\mathrm{CDH}$ : Congenital diaphragmatic hernia; CT: Computed tomography; IM: Intestinal malrotation; PPH: Persistent pulmonary hypertension; SMA: Superior mesenteric artery; SMV: Superior mesenteric vein; 3D: Three-dimensional.}

\section{Acknowledgements}

We would like to thank Enago (www.enago.jp) for English language review.

\section{Authors' contributions}

NE and KY contributed to the conception and design of the study. All authors contributed to the acquisition and analysis of data. NE and KY were major contributors in writing the manuscript. NE, KY, DK, SY, HW, KN, NT, TK, and NK were involved in treating the patient. All authors read and approved the final manuscript.

\section{Funding}

No grant support or funding from public institutions or private enterprises was received for this case report.

\section{Availability of data and materials}

The data sets analyzed during the current study are not publicly available due to their containing information that could compromise the privacy of research participants but are available from the corresponding author on reasonable request.

\section{Declarations}

Ethics approval and consent to participate

This article satisfied the consensus of the National Center for Global Health and Medicine Research Ethics Committee/Institutional Review Board.

\section{Consent for publication}

Informed consent was obtained from the patient presented in this article.

\section{Competing interests}

The authors declare that they have no competing interests.

Received: 8 March 2021 Accepted: 9 June 2021

Published online: 17 July 2021

\section{References}

1. Salaçin S, Alper B, Cekin N, Gülmen MK. Bochdalek hernia in adulthood: a review and an autopsy case report. J Forensic Sci . 1994;39:1112-6.

2. Kanazawa A, Yoshioka Y, Inoi O, Murase J, Kinoshita H. Acute respiratory failure caused by an incarcerated right-sided adult Bochdalek hernia: report of a case. Surg Today. 2002;32:812-5.

3. Thomas S, Kapur B. Adult Bochdalek hernia-clinical features, management and results of treatment. Jpn J Surg . 1991;21:114-9.

4. Loukas M, El-Sedfy A, Shane Tubbs R, Gribben WB, Shoja MM, Cermakova A. Vincent Alexander Bochdalek (1801-1883). World J Surg. 2008;32:2324-6.

5. Cannon C, Dildy GA, Ward R, Varner MW, Dudley DJ. A population-based study of congenital diaphragmatic hernia in Utah: 1988-1994. Obstet Gynecol . 1996;87:959-63.

6. Gedik E, Tuncer MC, Onat S, Avci A, Tacyildiz I, Bac B. A review of Morgagni and Bochdalek hernias in adults. Folia Morphol (Warsz). 2011;70:5-12.

7. Mullins ME, Stein J, Saini SS, Mueller PR. Prevalence of incidental Bochdalek's hernia in a large adult population. Am J Roentgenol. 2001;177:363-6.

8. Ohtsuka Y, Suzuki TH. Right-sided Bochdalek hernia in an elderly patient: a case review of adult Bochdalek hernias from 1982 to 2015 in Japan. Acute Med Surg. 2017:4:209-12.

9. Zenda T, Kaizaki C, Mori Y, Miyamoto S, Horichi Y, Nakashima A. Adult right-sided bochdalek hernia facilitated by coexistent hepatic hypoplasia. Abdom Imaging. 2000;25:394-6.

10. McVay MR, Kokoska ER, Jackson RJ, Smith SD. The changing spectrum of intestinal malrotation: diagnosis and management. Am J Surg. 2007;194:712-9.

11. Strouse PJ. Disorders of intestinal rotation and fixation ("malrotation"). Pediatr Radiol. 2004;34:837-51.

12. Salústio R, Nabais C, Paredes B, Sousa FV, Porto E, Fradique C. Association of intestinal malrotation and Bochdalek hernia in an adult: a case report. BMC Res Notes. 2014;7:1-4.

13. Machado NO. Laparoscopic repair of Bochdalek diaphragmatic Hernia in Adults. N Am J Med Sci. 2016;8:65-74.

14. Kocakusak A, Arikan S, Senturk O, Yucel AF. Bochdalek's hernia in an adult with colon necrosis. Hernia. 2005;9:284-7.

15. Niwa T, Nakamura A, Kato T, Kutsuna T, Tonegawa K, Kawai A, et al. An adult case of Bochdalek hernia complicated with hemothorax. Respiration. 2003;70:644-6.

16. Kubota K, Yamaguchi H, Kawahara M, Kaminishi M. Bochodalek hernia in a young adult: Report of a case. Surg Today. 2001;31:322-4.

17. Goh BKP, Teo MCC, Chng SP, Soo KC. Right-sided Bochdalek's hernia in an adult. Am J Surg. 2007;194:390-1.

18. Yamaguchi M, Kuwano H, Hashizume M, Sugio K, Sugimachi K, Hyoudou Y. Thoracoscopic treatment of Bochdalek hernia in the adult: report of 
a case. Ann Thorac Cardiovasc Surg Off J Assoc Thorac Cardiovasc Surg Asia. 2002;8:106-8.

19. Jambhekar A, Robinson S, Housman B, Nguyen J, Gu K, Nakhamiyayev $\checkmark$. Robotic repair of a right-sided Bochdalek hernia: a case report and literature review. J Robot Surg. 2018;12:351-5.

20. Rosen MJ, Ponsky L, Schilz R. Laparoscopic retroperitoneal repair of a right-sided Bochdalek hernia. Hernia. 2007;11:185-8.

21. Laaksonen E, Silvasti S, Hakala T. Right-sided Bochdalek hernia in an adult: a case report. J Med Case Rep. 2009:3:2-4.

22. Fraser JD, Craft RO, Harold KL, Jaroszewski DE. Minimally invasive repair of a congenital right-sided diaphragmatic hernia in an adult. Surg Laparosc Endosc Percutaneous Tech. 2009;19:5-7.

23. Patle NM, Tantia O, Prasad P, Das PC, Khanna S. Laparoscopic repair of right sided bochdalek hernia-a case report. Indian J Surg. 2013;75:303-4.

24. da Costa KM, Saxena AK. Congenital diaphragmatic hernia repair analysis in relation to postoperative abdominal compartment syndrome and delayed abdominal closure. Updates Surg. 2021. https://doi.org/10.1007/ s13304-021-00980-1.

25. Ito M, Terui K, Nagata K, Yamoto M, Shiraishi M, Okuyama H, et al. Clinical guidelines for the treatment of congenital diaphragmatic hernia. Pediatr Int Australia. 2021:63:371-90.

26. Iocono JA, Cilley RE, Mauger DT, Krummel TM, Dillon PW. Postnatal pulmonary hypertension after repair of congenital diaphragmatic hernia: predicting risk and outcome. J Pediatr Surg United States. 1999;34:349-53.
27. Marescaux J, Clément JM, Tassetti V, Koehl C, Cotin S, Russier Y, et al. Virtual reality applied to hepatic surgery simulation: the next revolution. Ann Surg. 1998:228:627-34.

28. Mise Y, Tani K, Aoki T, Sakamoto Y, Hasegawa K, Sugawara Y, et al. Virtual liver resection: computer-assisted operation planning using a three-dimensional liver representation. J Hepatobiliary Pancreat Sci. 2013;20:157-64.

29. Torjesen I. New 3D virtual human model aims to transform surgical training. BMJ. 2015. https://doi.org/10.1136/bmj.h6071.

30. Kenngott HG, Wagner M, Nickel F, Wekerle AL, Preukschas A, Apitz M, et al. Computer-assisted abdominal surgery: new technologies. Langenbeck's Arch Surg . 2015:400:273-81.

31. Barsom EZ, Graafland M, Schijven MP. Systematic review on the effectiveness of augmented reality applications in medical training. Surg Endosc. 2016;30:4174-83.

32. Pfeiffer $M$, Kenngott $H$, Preukschas $A$, Huber $M$, Bettscheider $L$, MüllerStich B, et al. IMHOTEP: virtual reality framework for surgical applications. Int J Comput Assist Radiol Surg. 2018;13:741-8.

\section{Publisher's Note}

Springer Nature remains neutral with regard to jurisdictional claims in published maps and institutional affiliations.

\section{Submit your manuscript to a SpringerOpen ${ }^{\circ}$ journal and benefit from:}

- Convenient online submission

Rigorous peer review

- Open access: articles freely available online

- High visibility within the field

- Retaining the copyright to your article

Submit your next manuscript at springeropen.com 